\title{
Revolutionizing Tree Management via Intelligent Spatial Techniques
}

\author{
Yiqun Xie, Shashi Shekhar \\ \{xiexx347,shekhar\}@umn.edu \\ Dept. of Computer Sci. and Eng. \\ University of Minnesota - Twin Cities
}

\author{
Richard Feiock \\ rfeiock@fsu.edu \\ Askew Sch. of Public Admin. \& Policy \\ Florida State University
}

\author{
Joseph Knight \\ jknight@umn.edu \\ Dept. of Forest Resources \\ University of Minnesota - Twin Cities
}

\begin{abstract}
Tree management is becoming a big issue in a variety of societal domains. In recent years, historic wildfires and blackouts caused by failures in tree management have increased in both quantity and severity, resulting in many deaths and financial loses in the tens of billions of dollars. Many communities are also suffering from massive tree loss (e.g., in the millions) that affects the health and well-being of citizens. These problems are likely to worsen due to climate change, aging infrastructure and population growth. Tree management needs a revolution to deal with these urgent problems. This opens up new challenges and opportunities for the spatial community. This paper presents some of the open research problems from the perspectives of individual tree mapping and characterization as well as decision making and in-field intervention.
\end{abstract}

\section{CCS CONCEPTS}

-Information systems $\rightarrow$ Spatial-temporal systems; Data mining; • Computing methodologies $\rightarrow$ Artificial intelligence.

\section{KEYWORDS}

Tree management; Spatial; Intelligent techniques; Vision

\section{ACM Reference Format:}

Yiqun Xie, Shashi Shekhar, Richard Feiock, and Joseph Knight. 2019. Revolutionizing Tree Management via Intelligent Spatial Techniques. In 27th ACM SIGSPATIAL International Conference on Advances in Geographic Information Systems (SIGSPATIAL '19), November 5-8, 2019, Chicago, IL, USA. ACM, New York, NY, USA, 4 pages. https://doi.org/10.1145/3347146.3359066

\section{INTRODUCTION}

In both urban and rural areas, trees play a huge role in the everyday life of humans. Knowing the locations of individual trees, their geometric characteristics (e.g., heights, canopy sizes), species and status (e.g., health) is critical to the resilience of many infrastructures (e.g., energy) and citizens' safety and well-being.

\subsection{Societal Significance and Urgency}

1.1.1 Resilience of Energy Infrastructure. Trees near electricity power lines pose major threats to energy infrastructure security,

Permission to make digital or hard copies of all or part of this work for personal or classroom use is granted without fee provided that copies are not made or distributed for profit or commercial advantage and that copies bear this notice and the full citation on the first page. Copyrights for components of this work owned by others than ACM must be honored. Abstracting with credit is permitted. To copy otherwise, or republish, to post on servers or to redistribute to lists, requires prior specific permission and/or a fee. Request permissions from permissions@acm.org.

SIGSPATIAL '19, November 5-8, 2019, Chicago, IL, USA

(c) 2019 Association for Computing Machinery.

ACM ISBN 978-1-4503-6909-1/19/11 . \$15.00

https://doi.org/10.1145/3347146.3359066 especially in the face of climate change. The core challenge faced by most communities under these threats is the lack of awareness of the location, type and wind resistance of trees relative to power line infrastructure in cities. For example, the 2003 Northeast Blackout, which was caused by unmanaged trees falling on power lines and subsequent cascade, affected over 50 million people. Such threats will likely worsen in the future due to climate change effects, aging infrastructure, and rapid population growth in cities.

In general, fallen or untrimmed trees are responsible for a significant portion of power outages (e.g., about $67 \%$ for DTE Energy [1]). High winds from severe storms can knock out trees, resulting in snapped power lines and blackouts that shut down hospitals, stores and businesses for extended periods. Such events are becoming increasingly frequent in many geographic regions (e.g., across the Atlantic and Gulf of Mexico coast). Recent hurricanes in Florida such as Hermine, Irma and Michael have exposed weaknesses in how well prepared a city is to operate and respond during and following catastrophic storms. Fallen trees caused long term electricity outages, road closures, service disruptions and loss of lives. In 2018, the power outage in Tallahassee (FL, USA) caused by broken power lines during Hurricane Michael affected $97 \%$ of the city's electric utility customers [11]. In Puerto Rico, a "single" fallen tree cut the main power-line and led to a power blackout for 900,000 customers as well as social outrage [3].

Trees near power lines have also caused many devastating wild fires in recent years. In California, the inability of effectively locating and trimming trees caused a series of deadly fires in 2018, including the Camp Fire, the deadliest wild fire in California history. The fires killed many people and destroyed over 10,000 built structures (e.g., homes) worth billions of dollars [6]. Smoke and unhealthy air that spread to cover over 20 cities caused schools to close for a week and severely hampered people's daily activities.

1.1.2 Resilience of Green Infrastructure. While trees growing in undesired locations (e.g., near power lines) can pose severe threats, in general they are necessary and invaluable components of our living areas. Trees purify the air, reduce urban heat island effects, create an aesthetically beautiful environment and promote mental health. However, many communities are facing a drastic loss of trees due to the global-spread of pests and disease (e.g., emerald ash borer, pine beetle, dutch elm disease, oak wilt). For example, the emerald ash borer, an invasive insect species, has expanded to 35 US states and killed millions of ash trees [2]. This can lead to severe environmental problems since ash trees cover $20-30 \%$ of treescapes in the majority of urban areas across the US. The cost of locating, treating or removing these ash trees has been estimated to be over 10 billion US dollars in the US alone. Another study found that the tree cover in US has declined in metropolitan areas across 45 states, 
resulting in an annual net loss of 36 million trees [8]. The same problem also exists in many other geographic areas. For example, ash trees in Europe are also facing extinction [4].

\subsection{Vision}

For resilient and sustainable communities, we envision the use of innovative spatial techniques to map the locations, characteristics and conditions of individual trees, and link this information to the locations of street and power line infrastructure to improve resilience to severe weather events in cities.

Currently, tree inventories only exist in very limited size and in very few geographic regions, because manual collection of information about individual trees is extraordinarily time-consuming and difficult (e.g., blocked GPS signal under tree canopies, hardto-measure canopy sizes, requiring expert knowledge to know the conditions or species).

Even utility companies, who regularly deal with vegetation management, still rely on human workers in vehicles and helicopters to check tree conditions with their eyes, a hit or miss solution that is both time-consuming and expensive. Additionally, the alarms raised by recent extreme weather events (e.g., storm, drought) and tree-related disasters (e.g., blackouts, fires) have prompted revisions in tree management regulations in many states for more aggressive and robust tree-trimming. While utilities already spend huge amounts of money every year on trimming tree branches and removing dead trees away from power lines, this makes it an even bigger issue. With the current technology, it has been estimated that even a slight change in tree-trimming regulations could easily cost a single utility hundreds of million dollars in extra per year. For example, utilities companies in Missouri estimated that tree trimming cost about 300 million dollars every year to meet the state's new vegetation management regulations [12]. Another study found that non-timely tree trimming can further increase costs by $20 \%$ to $70 \%$ [5]. In 2018, PG\&E, the largest utility company in California, was forced to file for bankruptcy due to the loss of lives and property damage in the billions associated with the forest fires started by trees near its power lines [6].

Innovations and advancements in spatial techniques (e.g., remote sensing, spatial data mining and machine learning) are necessary to address these urgent problems faced by communities. In the rest of the paper, we will detail the challenges and opportunities around three research areas that are needed to achieve our vision: (1) geometric profiling of individual trees, (2) determining species of individual trees, and (3) decision making and in-field management.

\section{CHALLENGES AND OPPORTUNITIES}

In order to generate informative inventories of individual trees to support decision-making and in-field intervention, we split the task into three research areas in a sequential order in Sec. 2.1 to 2.3. For each research area, we discuss existing techniques, identify research gaps and envision new opportunities.

\subsection{Geometric Profiling of Individual Trees}

First, we need to be able to detect the spatial locations of individual trees and their geometric characteristics, including heights, canopy sizes, shapes, etc. To achieve tree detection at a real-world large scales (e.g., city or state level), remote sensing data are typically required. There are two types of remote sensing data related to individual tree detection: satellite / aerial imagery and topographic models (e.g., digital surface models, LiDAR point cloud). The main difference between the two is that satellite / aerial imagery records the spectral value (e.g., intensity of a color channel) of each location whereas topographic models record the canopy height (e.g., height of a building roof or a tree canopy). Fig. 1 (a) and (b) show examples of the two data types for the same geographic region.

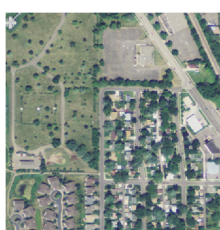

(a) Imagery

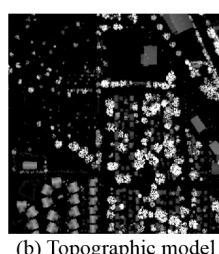

(b) Topographic model

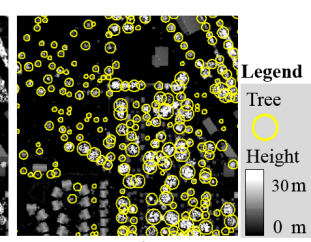

(c) Detections
Figure 1: Remote sensing data for tree detection [15].

Compared to imagery, topographic models have two major advantages: (1) Tree boundaries are easier to separate using geometric characteristics (i.e., change of heights). This can be very difficult with imagery where nested groups of trees often share very similar spectral information (e.g., colors), making their boundaries hard to recognize; (2) Tree features are more stable and homogeneous in topographic models since they are not affected by changes in brightness, radiation, camera angles, etc. As a result, most existing work is based on topographical models and applies geometric segmentation methods (e.g., watershed segmentation) to split groups of trees into individual trees (e.g., [7]). However, these methods are mostly designed for forest regions where the landscapes are assumed to be homogeneous of trees only, and cannot be applied in urban areas where the environment is a complex mixture of different types of objects. In addition, these unsupervised segmentation methods often generate fixed partitions that tend to favor local minima and split a single tree into multiple pieces [15]. Deep learning methods have achieved promising success in everyday object detection (e.g., YOLO [9], SSD, r-CNN), but they require a huge amount of training data (i.e., manually annotated tree objects) which is not available in vast majority of the geographic areas. In addition, our previous study [15] showed that deep learning based object detectors still have difficulty in separating out individual trees even with reasonable training data in a local area.

To address these limitations, our earlier work [15] proposed a TIMBER framework to detect individual trees in both urban and forest environments using a combination of geometric optimization (unsupervised) and a deep learning based filter (supervised). The geometric optimization phase uses "dome-shaped" mathematical approximators (e.g., Gaussian) to approximate the real geometric shapes of trees in topographic models. The decision variables in the optimization formulation are the locations, heights and sizes of treelike structures. Since tree surfaces are not smooth and contain many dome-shaped local bumps, we designed specific regularizers to avoid such noise in the result. The output of geometric optimization contains locations, heights and sizes of all tree-like structures (i.e., dome-shaped trees and non-trees). To remove non-tree structures, we constructed a convolutional neural network based filter, which 
learns and predicts a binary class label (i.e., tree and non-tree) for each tree-like structure. The deep learning filter is only used as a classifier, not a direct object detector, which avoids the difficulty of boundary separation. Fig. 1 (c) shows an example result of TIMBER.

While TIMBER was able to greatly improve tree detection performance on topographic models, two main challenges and opportunities need to be further explored for tree management applications.

First, compared to satellite / aerial imagery, current topographic models have a relatively long update period (e.g., five years). This is not timely for tree inspection and management which needs to be done at least once a year. We envision a heterogeneous-source based incremental-update framework to address this challenge. The framework has two phases: (1) a base phase: tree detection using TIMBER on topographic models; and (2) an update phase: incrementally modify and update detection results by tracking local changes in satellite / aerial imagery. The framework can potentially take advantage of both types of data, where the topographic models contain stable geometric features and the imagery has a higher frequency of update (e.g., monthly). In addition, tracking local changes (e.g., tree growth or removal) in the imagery for each detected individual tree is potentially a much easier task than directly detecting and separating nested trees in the imagery.

Second, the current mathematical approximators (e.g., truncated Gaussian with varying parameters) in TIMBER may not be suitable for all tree species. Since TIMBER has only been tested in Minnesota, it may not cover certain tree species (e.g., palm trees) or landscapes (e.g., heavily overlapped tree-tops in tropical forests) that are specific to other geographic regions. Further tests and explorations of new approximators are needed to improve its flexibility and geographic robustness.

\subsection{Determining Species of Individual Trees}

Tree species provide meaningful information in tree management. For example, for energy infrastructure security, tree properties (e.g., wind resistance) determined by species or genera are critical for risk analysis (e.g., blackouts, fires) involving trees and power lines.

With detections of individual trees (Sec. 2.1), this step can focus on feature identification / construction and species classification for each individual tree. In general, tree species classification is considered one of the most challenging problems in remote sensing. In satellite imagery with visual bands, trees of different species often appear as very similar green blobs and have very little distinction [14]. In related work, most promising results are achieved with hyperspectral imagery. Compared to visual-band based satellite or aerial imagery, hyperspectral imagery contains values for a significantly greater number of spectral bands (e.g., hundreds). With such rich information, a few studies have demonstrated the feasibility of identifying distinctive signatures of several species. As the saying goes, however, there is no free lunch. Hyperspectral imagery is very expensive to capture at high resolution (e.g., meter or sub-meter level) so it is still unavailable at large scales (e.g., city or state level) in very most of the urban areas. The previous studies were mainly performed in a few controlled local areas where the imagery was specifically collected. Topographic models face a similar problem. Since topographic models only have the height information and do not contain the rich spectral details in hyperspectral imagery, their spatial resolution has to be much higher (e.g., centimeter level) to capture detailed structural differences between different species. However, due to cost concerns, existing topographic models mostly have their resolution at meter level, which is sufficient for individual tree detection but not for species classification.

Currently, the only remote sensing data that have centimeter level resolution and are collected regularly at large scales (e.g., city scale or greater) is visual-band based aerial imagery. For example, a large number of counties have aerial imagery with 4-7 $\mathrm{cm}$ resolution on an annual or sub-annual basis. These datasets are for general purposes and are often collected during leaf-off season (e.g., winter, spring) to reduce canopy coverage on the other objects. While the absence of leaves makes it even more difficult to extract signatures of different species directly from the tree canopies, our preliminary study takes advantage of this and identifies a side-feature - tree shadow - that shows encouraging results on species and genus classification. Fig. 2(a) shows a typical example of aerial imagery. With leaf-off, we can see the detailed branch structures of trees through their shadows. In fact, even with leaf-on the tree profile structures from the shadows often contain more unique characteristics than the canopies from top-down views. Fig. 2(b) shows examples of shadows from different species of trees, which were clipped from aerial imagery. As we can see, different species do exhibit different shadow signatures. This new finding opens up new opportunities in tree species and genus classification at real-world large scales. Fig. 2(c) shows the preliminary classification results. The training set (about 2000 samples) and test areas contain 24 different classes. The green boxes show the test samples (a subset of trees in an area that was not used in training) that have the correct tree label in the top 3 predictions of the convolution neural network and red boxes not. Note that this is a very preliminary result and is just used to demonstrate the potential feasibility of this envisioned methodology (not matured work).

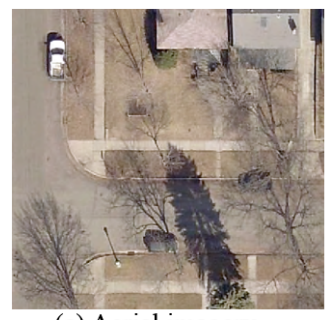

(a) Aerial imagery

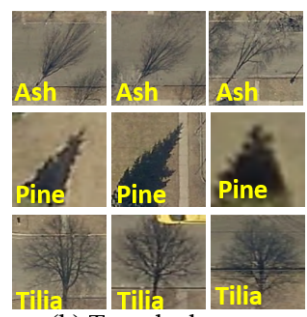

(b) Tree shadows

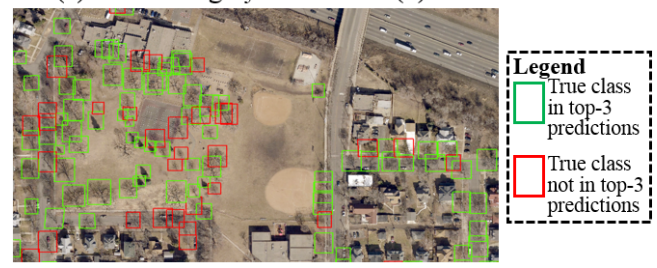

(c) Preliminary results on several test samples

Figure 2: Opportunity: species classification using shadows.

A major challenge in this method is generating the training data. While it is relatively easy to generate the bounding boxes of shadows using the geometric properties of individual trees (Sec. 2.1) and sun angle information from the imagery, it is challenging to label the species or genera. Citizen science platforms (e.g., Zooniverse, 
Amazon Mechanical Turk) provide a potential solution. However, unlike everyday objects, expert knowledge on trees is required to recognize their species or genera, which may not be a trivial task. To address this issue, future work may borrow experience from other domains (e.g., Galaxy Zoo) which have pre-training schemes to assist participants with manual classification.

Another challenge is the overlaps between shadows and other objects (e.g., buildings) as well as the shadows themselves. We can often solve shadow-object overlaps using multiple aerial images taken at different hours (e.g., morning, afternoon) so that shadows cast onto buildings (i.e., blurry) in one may be on streets (i.e., clearer) in another. Shadow-shadow overlaps may require more sophisticated methods to handle. One potential solution might be to add combined labels (e.g., "ash and elm") to overlapped regions and postprocessing steps to separate the predictions into individual species or genera. Capsule networks [10], a type of convolutional neural network that was designed for segmenting overlapping objects (e.g., digits), can also be explored to tackle this issue.

Certain geographic regions can also be more challenging for shadow-based species classifiers. For example, trees in regions with a tropical climate can be ever-green, making it more difficult to see the differences in branch structures of trees in their shadows. Thus, we also envision other research directions which may be more suitable for those regions. First, future research can take advantage of tree phenology and explore new visual-band based features using temporal or seasonal changes in tree characteristics. The idea is that trees of different species may change color, flower or wither at different time. This temporal variation may lead to visibly distinct signatures of different species in aerial imagery. Second, we can reduce the difficulty of the problem based on specific application needs. For example, if we are mainly interested in finding species of low-wind resistance, we may simplify the problem by grouping the species or genus into a much smaller number of classes. Other properties (e.g., branch thickness, tree age) may also be good indicators of wind resistance.

\subsection{Decision Making and In-field Management}

With a map of individual trees and their properties, another challenge and opportunity is to assist the decision making process and in-field interventions. For decision making, an important first step is data integration (e.g., tree databases and power line databases). New conceptual and logical data models, SQL clauses and predicates may be needed to support efficient querying in an integrated database. We can also leverage spatial data mining techniques to facilitate risk analysis and management plan optimization. For example, spatial buffer or hotspot analysis can help identify risky spots where trees may fall onto power lines in storms. Spatial predictive models can then be used to better quantify the risk using the strength of a storm and use optimization methods to prioritize tree trimming zones under a budget limit. We also need new spatial data mining techniques to incorporate uncertainty or statistical measures to model the errors from tree detection and classification phases (e.g., data aging, detection inaccuracy).

Assistance to in-field interventions may leverage recent advancements in robotics. In recent years, specialized drone assistants have been developed for forest fire containment [13], in-field sensor deployment, etc. For example, drones can easily carry sensors to regions that are difficult and time-consuming to reach by humans (e.g., forested areas) and install them at the desired locations efficiently. Future specialized drones may carry trimming tools to cut smaller branches of trees near power lines or put a clear mark on those trees so that in-field workers can easily identify trees that need trimming.

\section{CONCLUSIONS}

We propose a vision for the next generation of tree management using innovative spatial techniques. Smart tree management is both important and urgent due to the tree-related problems (e.g., historical fires, blackouts, loss of green areas) that have increased in frequency, scale and severity. This vision cannot be fully realized without significant advancements in individual tree detection and characterization using heterogeneous sources of spatial data. We encourage the spatial community to explore these challenges and opportunities and revolutionize tree management to improve sustainability, safety and well-being.

\section{ACKNOWLEDGMENTS}

This work is supported by the US NSF under Grants No. 1901099, 1737633, 1541876, 1029711, IIS-1320580, 0940818 and IIS-1218168, the USDOD under Grants HM0210-13-1-0005, USDA under Grant No. 2017-51181-27222, ARPA-E under Grant No. DE-AR0000795, NIH under Grant No. UL1 TR002494, KL2 TR002492 and TL1 TR002493, and the OVPR U-Spatial and Minnesota Supercomputing Institute at the University of Minnesota. We also thank Sam Detor and Abigail Roh for their help on data generation and evaluation.

\section{REFERENCES}

[1] 2018. DTE Energy. https:/www.newlook.dteenergy.com/wps/wcm/connect/ dte-web/home/service-request/common/system-improvements/tree-trimming.

[2] 2018. Emerald Ash Borer. https://www.nrs.fs.fed.us/disturbance/invasive_ species/eab/effects impacts/cost of infestation/.

[3] Nicole Acevedo. 2018. Puerto Rico: Single fallen tree on power line leaves 900K without power. NBC News. https://www.nbcnews.com/storyline/puerto-ricocrisis/puerto-rico-fallen-tree-power-line-leaves-900k-without-power-n865506.

[4] BBC News. 2016. Ash tree set for extinction in Europe. http://www.bbc.com/news/science-environment-35876621.

[5] D Mark Browning. 1997. The economic impacts of deferring electric utility tree maintenance. Journal of Arboriculture 23 (1997), 106-112.

[6] U. Irfan. 2018. California's largest utility just declared bankruptcy. Hello, climate change. https://www.vox.com/2019/1/14/18182162/pg-e-camp-fire-bankruptcy.

[7] Matti Maltamo et al. 2009. Predicting tree attributes and quality characteristics of Scots pine using airborne laser scanning data. SILVA FENNICA 43, 3 (2009).

[8] David J Nowak and Eric J Greenfield. 2018. Declining urban and community tree cover in the United States. Urban forestry \& urban greening 32 (2018), 32-55.

[9] Joseph Redmon, Santosh Divvala, et al. 2016. You only look once: Unified, realtime object detection. In Proceedings of the IEEE conference on computer vision and pattern recognition. 779-788.

[10] Sara Sabour et al. 2017. Dynamic routing between capsules. In Advances in neural information processing systems. 3856-3866.

[11] Jeffrey Schweers. 2018. Hurricane Michael: Nearly 114,000 without power this morning. https://www.tallahassee.com/story/news/2018/10/10/power-outagesgrow-hurricane-michael-approaches-high-winds/1591767002/.

[12] The Joplin Globe. 2018. Missouri: State board trims back proposed tree-trimming rule. https:/www.joplinglobe.com/archives/ missouri-state-board-trims-back-proposed-tree-trimming-rule/article_ e6a3d909-6507-565a-87b4-2d28d732805c.html.

[13] Dirac Twidwell, Craig R Allen, Carrick Detweiler, James Higgins, Christian Laney, and Sebastian Elbaum. 2016. Smokey comes of age: unmanned aerial systems for fire management. Frontiers in Ecology and the Environment 14, 6 (2016), 333-339.

[14] Yiqun Xie et al. 2018. Transforming Smart Cities with Spatial Computing. In 2018 IEEE International Smart Cities Conference (ISC2). IEEE, 1-9.

[15] Yiqun Xie, Han Bao, Shashi Shekhar, and Joseph Knight. 2018. A TIMBER Framework for Mining Urban Tree Inventories Using Remote Sensing Datasets. In 2018 IEEE International Conference on Data Mining (ICDM). IEEE, 1344-1349. 